\title{
Vaccine-Associated Paralytic Poliomyelitis
}

National Cancer Institute

\section{Source}

National Cancer Institute. Vaccine-Associated Paralytic Poliomyelitis. NCI Thesaurus. Code C113671.

A rare, paralytic poliomyelitis associated with the orally administered live attenuated strain of the poliovirus, OPV. 